\title{
Der Stakeholderdialog zwischen Regulierung und Rhetorik \\ Eine empirische Studie der dargestellten Dialogorientierung in deut- schen und dänischen Geschäftsberichten
}

\author{
Anne Grethe J. Pedersen ${ }^{* *}$
}

\section{Stakeholder Dialogue between Regulation and Rhetoric}

Stakeholder dialogues are part of Corporate Governance Regulations and therefore a management instrument that many companies deal with and communicate about. This article investigates how German and Danish chemical companies present and construct dialogue with stakeholders in their annual reports. This entails analyzing, first, to what extent stakeholder dialogue is articulated, second how the stakeholder dialogue is evaluated linguistically and, third, what approaches to stakeholder dialogue are expressed in the reports. Moreover, I address some possibilities for better communication about stakeholder dialogue as a means of differentiation and corporate identity profiling in relation to competitors.

Keywords: Stakeholder dialogue, annual report, Corporate Governance, Corporate Social Responsibility

\section{Einleitung}

Der vorliegende Beitrag beschäftigt sich mit der Kommunikation über Stakeholderdialoge in Geschäftsberichten. Dabei wird konkret die Darstellung des Stakeholderdialogs in deutschen und dänischen Geschäftsberichten aus sprachwissenschaftlicher Perspektive untersucht und diskutiert. Hierbei wird auf Verbesserungsmöglichkeiten und ungenutzte Potenziale in der Darstellung des Stakeholderdialogs aufmerksam gemacht. Die Darstellung des Stakeholderdialogs gehört zu den nicht-finanziellen, image- und vertrauensbildenden Teilen von Geschäftsberichten, die nicht selten unter dem Vorwurf leiden, „Austauschbarkeiten“ bzw. Allgemeinplätze zu vermitteln. Damit ist gemeint, dass die Texte oft allgemein, unverbindlich, zeitlos und beliebig sind (vgl. Bextermöller 2006: 91) und dass sich die vermittelten Corporate Identities und Corporate $V$ alues nicht deutlich genug voneinander unterscheiden, um beim Leser einen klaren Eindruck von ihrer unternehmens-spezifischen Einzigartigkeit zu hinterlassen. Beispielsweise werden Unternehmenswerte wie Glaubwürdigkeit, Offenheit und Dialogbereitschaft sehr häufig aufgeführt, aber solche Werte haben einen allgemein plausiblen Charakter, so dass kein Unternehmen das Gegenteil von sich behaupten würde. Kein Unternehmen würde für sich in Anspruch nehmen, unglaubwürdig und nicht zum Dialog mit zentralen Anspruchsgruppen bereit zu sein. Die getroffenen Aussagen verlieren deshalb an Wert im Sinne einer Profilierung und einer Differenzierung ge-

Beitrag eingereicht am 15.04.2011: nach doppelt verdecktem Gutachterverfahren überarbeitete Fassung angenommen am 17.04.2011.

** Dr. Anne Grethe J. Pedersen, Institut für Kultur und Globale Studien, Aalborg Universität, Kroghstraede 3, DK-9220 Aalborg Ost, Tel.: +45 9940 9187, Fax: +45 9815 2304, E-Mail: agp@cgs.aau.dk, Forschungsschwerpunkte: Wirtschaftskommunikation, Sprache und Kultur. 
genüber Konkurrenten am (Finanz-) Markt. Wohlwissend, dass Ausnahmen existieren, ist der Ausgangspunkt dieses Beitrags, dass in der Unternehmenskommunikation ein Bedarf an einer stärker differenzierten Selbstdarstellung besteht und dass die Darstellung des Stakeholderdialogs hierfür eine geeignete Möglichkeit bietet.

Mit dem Ziel, zu prüfen, wie bestimmte Verhaltensregulierungen im Bereich der Corporate Governance in der Unternehmenskommunikation berücksichtigt werden und inwieweit diese Berücksichtigung zur differenzierten Unternehmensidentität beiträgt, wird folgenden Forschungsfragen nachgegangen:

In welchem Umfang und auf welche Art werden Stakeholderdialoge in Geschäftsberichten thematisiert und dargestellt?

Welche Ansätze zu den Begriffen Stakeholder und Stakeholderdialog werden dargestellt?

Inwieweit bleiben Potenziale zur Corporate Identity-Vermittlung offen?

Es wird von der Hypothese ausgegangen, dass mit der Homogenität von Geschäftsberichten das Potenzial zur Profilierung von Corporate Identity und Differenzierung von Wettbewerbern verloren geht.

\section{Stakeholderdialog als Instrument der Unternehmenskommunikation}

Der zunehmende Fokus auf Nachhaltigkeit, Unternehmensethik, Corporate Social Responsibility, Corporate Citizenship ${ }^{1}$, und ähnliche Begriffe (vgl. Bassen et al. 2005; Lindgreen/Swaen 2010) hat die Notwendigkeit des Dialogs zwischen Unternehmen und ihren Stakeholdern bestärkt. In ihrem Grünbuch Europäische Rabmenbedingungen für die soziale Verantwortung der Unternehmen stellte die EU-Kommission 2001 fest, dass „ein wichtiger Aspekt der CSR die Wechselbeziehungen zwischen den Unternehmen und ihren internen und externen Stakeholdern (Arbeitnehmern, Kunden, Nachbarn, nichtstaatlichen Organisationen, Behörden usw.) " sind (Kommission der Europäischen Gemeinschaften 2001: 7). Die genannten Wechselbeziehungen sind aus Unternehmenssicht deshalb wichtig, weil sie es ermöglichen, herauszufinden, welche konkreten Anforderungen und Erwartungen an das Unternehmen gestellt werden. Generell wird erwartet, dass Unternehmen als verantwortungsvolle Corporate Citizens agieren und Geld, Sachmittel, Zeit und/oder Wissen in die Lösung von sozialen und ökologischen Problemen wie beispielsweise dem Klimawandel und der Verletzung von Menschenrechten investieren (vgl. Braun 2008). Das unternehmerische Ziel der Gewinnmaximierung hat sich um soziale, ökologische und ethische Ziele erweitert. Zudem werden bei der Bewertung von Unternehmen durch z. B. Investoren und Finanzanalytiker immer öfter auch nicht-finanzielle Aspekte berücksichtigt, besonders bei den Socially Responsible Investments ${ }^{2}$. Diese Perspektive auf die Rolle der Unternehmen scheint seit

1 Vgl. die Typologie der Partnerschaftsformen und Instrumente des Corporate Citizenship (Dresewski und Koch 2011).

$2,[\ldots]$ an investment that combines an investor's financial objectives with his or her commitment to social concerns, such as peace, social justice, economic development, or healthy environment; an investment which may or may not have economic return as its principal goal, made with an in- 
Jahren die Debatte über Management und Unternehmensverhalten zu dominieren, steht aber nicht unumstritten da. Mit der Behauptung, dass das gesellschaftliche Engagement von Unternehmen nur zu steigenden Kosten und Preisen führe, weist u. a Henderson (2001) auf potenzielle Dilemmata der sozialen Verantwortung von Unternehmen hin. Diese Sichtweise ist heute allerdings eher die Ausnahme, was eine Vielfalt an CSR-Maßnahmen und CSR-Kommunikation sowie eine sehr breite Auswahl an CSR-Literatur (vgl. Morsing et al. 2006; May et al. 2007; Wieland 2007, Hiß 2009; Raupp et al. 2011) bestätigen.

Fragt man nach den Ursachen für das Verlangen nach unternehmerischer Mitverantwortung bei der Lösung von gesellschaftlichen Problemen, ergeben sich mehrere mögliche Antworten. Die zunehmende Fokussierung auf CSR hängt zum einen damit zusammen, dass Stakeholder eine bessere Einsicht in das Unternehmenshandeln bekommen, u. a. durch die Internetpräsenz der Unternehmen, aber auch durch die zunehmende Berichterstattung in Print und Online. Zum anderen sind viele der aktuellen Problemstellungen von globalem Charakter und erfordern globale Lösungen, die aber über die Kompetenzen der nationalen Regierungen hinausreichen. Im Gegensatz zu den nationalen Regierungen sind viele Unternehmen global tätig und können Entscheidungen beeinflussen, die sich außerhalb der Reichweite nationaler Politik befinden (vgl. Beck 1999, 2003; Brock 2008). Zum dritten bewirken ein allgemein erhöhtes Risikobewusstsein und die Besorgnis über Ressourcenmangel, Hungersnöte, Armut, Klimaveränderungen und Krankheiten in der Welt, dass sich die Erwartungen an Unternehmen verschärfen (vgl. Morsing 2005: 12; Beck 1999, 2003). Themen wie Umwelt, Ethik und Soziales erhalten große Aufmerksamkeit in den Medien, in der Politik und bei den Verbrauchern, die im Falle enttäuschter Erwartungen bestimmte Produkte boykottieren können. Durch die Auseinandersetzung mit diesen ständig wechselnden Themen kann das Unternehmen Vertrauen gewinnen, indem es signalisiert, dass es gegenüber Interessen und Anforderungen im gesellschaftlichen Umfeld offen ist, auch wenn diese mit den Interessen des Unternehmens vordergründig nicht im Einklang stehen ${ }^{3}$. Interessenkonflikte lassen sich nicht vermeiden, was auch der Leser eines Geschäftsberichts weiß. Deshalb kann es der Glaubwürdigkeit schaden, besonders relevante umstrittene Themen nicht $\mathrm{zu}$ thematisieren. Wenn bestimmte issues nicht thematisiert werden ${ }^{4}$, wird es dem Leser überlassen, sich eigene Annahmen über die Position des Unternehmens zu bilden, die nicht notwendigerweise zu Gunsten des Unternehmens ausfallen. Voraussetzung für die Thematisierung und Auseinandersetzung ist allerdings, dass das Unternehmen die Interessen der Stakeholder kennen lernt, z. B. durch einen Stakeholderdialog. Dies ist wiederum Voraussetzung dafür,

tent to take into account the impact of the investment on the society in which the investment is made“ (Kinder et al. 1992: 852).

3 Holmgreen (2008) zeigt beispielsweise, wie der Vorstandsvorsitzende eines dänischen BiotechUnternehmens in seinen Geschäftsberichten versucht, ein eher skeptisches gesellschaftliches Umfeld von den Chancen der Biotechnologie zu überzeugen, u.a. durch die Metaphern „,nature is technology" und „biotechnology is cleanliness“.

4 Larsen/Pedersen (2010) zeigen, dass sich nur 6 von insgesamt 63 deutschen und dänischen Fluggesellschaften auf ihren Websites mit Klimawandel und Klimaschutz auseinandersetzen, obwohl der Flugverkehr oft mit einer nicht unwesentlichen Klimabelastung assoziiert wird. 
dass einflussreiche Stakeholder das Unternehmen unterstützen und dass Risiken aufgrund möglicher kritischer Einflussgruppen frühzeitig erkannt und ggf. begrenzt werden können.

Wie im Grünbuch der EU-Kommission wird der Stakeholderdialog auch von anderen richtungsweisenden Institutionen hervorgehoben und empfohlen. Zum Beispiel schreibt der UN Global Compact ${ }^{5}$ auf seiner Website:

„A culture of dialogue and learning is crucial to continuous performance improvement. From its inception, the Global Compact has fostered and promoted dialogue between business and other stakeholders around critical challenges, covering a diverse range of sustainability issues." 6

Der durch den Stakeholderdialog ermöglichte Moment des Lernens ist in diesen Empfehlungen von zentraler Bedeutung. Eine (Unternehmens-) Kultur des Dialogs und des Lernens, die den Austausch von Erfahrungen, Wissen, Haltungen und Befindlichkeiten schätzt und praktiziert, ist für die erfolgreiche Entwicklung des Unternehmens entscheidend und somit vor allem für das Management ein nützliches decision makingInstrument ${ }^{7}$. Dieser Zusammenhang zwischen dem organisatorischen Lernen und dem Stakeholderdialog wird ebenso in Burchell und Cook (2008) behandelt und betont.

Darüber hinaus wird im deutschen und dänischen Corporate Governance Kodex (CGK) die Bedeutung des Stakeholderdialogs betont, im dänischen jedoch expliziter als im deutschen:

„Der Vorstand leitet das Unternehmen mit dem Ziel nachhaltiger Wertschöpfung in eigener Verantwortung und im Unternehmensinteresse, also unter Berücksichtigung der Belange der Aktionäre, seiner Arbeitnehmer und der sonstigen dem Unternehmen verbundenen Gruppen (Stakeholder)“ (Deutscher CGK 2009, Abschnitt 4.1.1).

„[...] it is desirable that the company's management run and develop the company with due consideration of its stakeholders, and that the management provide an incentive for dialogue with these stakeholders" (Dänischer CGK 2009 in englischer Übersetzung).

Die obigen Zitate machen deutlich, dass ein Unternehmen, das den Normen einer Good Corporate Governance nachkommen will, die Interessen seiner Stakeholder berücksichtigen und den Dialog mit ihnen suchen muss. Hierüber sollte dann auch berichtet werden. Für die 1.100 größten dänischen Unternehmen ist sogar gesetzlich vorgeschrieben, ab 2009 ihre Maßnahmen zur gesellschaftlichen Verantwortung im Ge-

5 Der im Jahre 2000 gegründete UN Global Compact ist ein freiwilliges Netzwerk mit dem Ziel, nachhaltige Märkte zu schaffen so wie das gesellschaftliche Engagement von Unternehmen zu fördern.

6 http://www.unglobalcompact.org/docs/news_events/8.1/GC_brochure_FINAL.pdf, Website aufgerufen am 27.05.2011.

7 Eine empirische Studie von 46 Unternehmen zeigt jedoch, dass der Einfluss der Stakeholder auf corprate decision making in vielen Unternehmen begrenzt ist: „In the majority of cases (25 in total) there was evidence of limited and intermediary impacts related to stakeholder engagement. In these cases stakeholder opinions for example impacted on corporate reporting or helped establish a materiality matrix" (Spitzeck/Hansen 2010: 382). 
schäftsbericht zu erläutern ${ }^{8}$. Diese Gesetzesanforderung hat laut der Website des dänischen Energiekonzerns Dong Energy ${ }^{9}$ internationale Anerkennung eingebracht und sei bis auf weiteres ohne Parallele in anderen Ländern.

Um ihre Existenz zu legitimieren und Vertrauen zu gewinnen, müssen Unternehmen auf Anforderungen und Erwartungen ihrer Stakeholder, etwa den Gesetzgeber, reagieren und ihre CSR-Maßnahmen glaubwürdig kommunizieren. Den obigen regulierenden Empfehlungen ist jedoch keine Präzisierung des Stakeholder-dialogkonzepts zu entnehmen, weshalb eine Klärung zentraler Aspekte des Stakeholderdialogs nützlich erscheint.

\subsection{Drei Ansätze zum Begriff des Stakeholderdialogs}

Um begriffliche Klarheit zu schaffen, sollte zunächst geklärt werden, was unter einem Stakeholder und einem Dialog zu verstehen ist. Danach wird auf drei unterschiedliche Ansätze des Stakeholderdialogs eingegangen, um die theoretische Grundlage für die empirische Analyse zu verdeutlichen.

\subsubsection{Der verwendete Stakeholderbegriff}

Ein Stakeholder ist nach dem englischen Ursprung des Wortes : „, person or a company that is involved in a particular organization, project, system, etc., especially because they have invested money in it" (Wehmeier 2000: 1260), d.h. jemand, der einen (Wett-) Einsatz, also ein (ökonomisches) Interesse an einem Unternehmen hat, und der riskiert, diesen Einsatz zu verlieren. Synonym verwendete Begriffe sind u. a Interessent und Anspruchsgruppe, aber allgemein gesehen hat sich der Stakeholderbegriff wie auch viele andere angloamerikanische Wörter in den Wortschatz des Wirtschaftslebens eingebürgert. Es werden drei grundlegend unterschiedliche Perspektiven auf die Bedeutung der Stakeholder für das Unternehmen und somit auf die Motivation für die Interaktion mit den Stakeholdern unterschieden. Im klassischen, instrumentellen Stakeholdermodell (vgl. Frederiksen et al. 2003: 111ff.; Spitzeck/Hansen 2010: 379ff.) haben alle Stakeholder ein ökonomisches Interesse am Unternehmen. Die einflussreichsten Stakeholder werden deshalb einbezogen und berücksichtigt, weil das Unternehmen ihre Unterstützung braucht, um seinen wirtschaftlichen Erfolg zu sichern. In diesem engeren Sinne beschränken sich die Stakeholder vorwiegend auf Kunden, Mitarbeiter, Aktionäre, Lieferanten, Kreditinstitute und Behörden. Die deskriptive Perspektive zielt allein auf die Identifizierung und Klassifizierung der Stakeholder einer Organisation ab, ohne die Legitimität der durch die jeweiligen Stakeholdergruppen vorgebrachten Ansprüche zu bewerten (vgl. Lozano 2005). Das normative Stakeholdermodell dagegen reflektiert eine nuanciertere und breitere Auffassung davon, wer die Stakeholder eines Unternehmens sind und welche Arten von Interessen sie vertreten. Nach diesem Ansatz sind das Unternehmen und die Stakeholder Teil eines Ressourcennetzwerks, in dem auch nicht-finanzielle Werte zur Geltung kommen. Die Anzahl der möglichen Stakeholder erhöht sich dadurch deutlich und umfasst bei-

8 Laut der im Dezember 2008 beschlossenen Gesetzesänderung: \99a des dänischen Gesetzes über den Jahresabschluss Arsregnskabsloven (siehe z.B. www.csrgov.dk/sw51190.asp).

$9 \quad$ www.dongenergy.dk/erhverv/klimamiljo/indsigtogviden/Pages/lovomsamfundsansv.aspx. 
spielsweise auch Nichtregierungsorganisationen und Nachbarn, deren Legitimität eher auf moralischen als ökonomischen Rechten basieren. Wenn es zum Dialog mit den Stakeholdern kommt, erfordert der normative Ansatz deshalb eine Einordnung der Stakeholder nach Wichtigkeit und Relevanz in der gegebenen Situation, weil die für einen Dialog notwendigen Ressourcen nicht unbegrenzt vorhanden sind (vgl. Frederiksen et al. 2003: 112ff.; Spitzeck/Hansen 2010: 380). In Anlehnung an den normativen Stakeholderbegriff werden in diesem Beitrag Stakeholder als Personen oder Gruppen von Personen inner- oder außerhalb der Organisation verstanden, die die Geschäftstätigkeit eines Unternehmens beeinflussen und/oder von ihr beeinflusst werden (vgl. Freeman 1984: 46; Frederiksen et al. 2003: 113; Bauhofer 2004: 32f.; Deix 2005).

\subsubsection{Das zugrunde gelegte Verständnis vom Dialog}

Eine nützliche, weil umfassende und präzisierende Definition des Dialogbegriffs ${ }^{10} \mathrm{im}$ organisationskommunikativen Kontext ist die von Bloch-Poulsen (2006):

„Ein Dialog ist ein gemeinsames, untersuchendes Gespräch über ein Thema, in dem im Voraus keine Antwort gegeben ist, in dem man Wissen und Erfahrungen teilt, in dem man bereit ist, die eigenen Annahmen und die der anderen infrage zu stellen, in dem man durch das Sprechen auf gleicher Augenhöhe dazu beiträgt, dass sich alle wohl fühlen, so dass man es wagt, sich auf dünnes Eis zu bewegen, in dem man eine Entscheidung gemeinsam treffen kann" (BlochPoulsen 2006: 9, eigene Übersetzung).

Demnach erfordert ein Dialog große Anstrengungen, Zeit, Kompetenz, Willen, Offenheit, Engagement und Kompromissbereitschaft. Es ist eine anspruchsvolle Dialogauffassung, wobei auch Schwächen offengelegt werden können. Man kann sich fragen, ob diese Kriterien alle gleichzeitig erfüllt werden können, wenn sich ein Unternehmen und dessen Stakeholder an einen Tisch setzen. Wahrscheinlich sind eine symmetrische Kommunikation auf gleicher Augenhöhe und das Treffen einer gemeinsamen Entscheidung selten realistisch, weil einer der Dialogpartner, in der Regel das Unternehmen, das Sagen hat. Etwas anderes ist speziell dann zu erwarten, wenn das Unternehmen in einer Krise steckt und es von der Bewertung der relevanten Stakeholder abhängig ist, um sein Image oder gar seinen Fortbestand zu retten. Auch wenn die hier verwendete Definition möglicherweise eine Idealvorstellung beschreibt, enthält sie doch sehr zentrale und grundlegende Elemente für einen wertvollen und zweckmäßi-

10 Burchell und Cook $(2006,2008)$ liefern empirische Daten zu den Dialogauffassungen verschiedener Dialogpartner und diskutieren die Komplexität und Diversität der Dialogdefinitionen sowie die Herausforderungen, die mit der Schaffung eines effektiven und bewertbaren Stakeholderdialogs verbunden sind. Van Huijstee und Glasbergen (2007) geben einen Einblick in verschiedene Aspekte der praktizierten Stakeholderdialoge zwischen multinationalen Unternehmen und NGOs über Umweltprobleme. Kaptein und van Tulder (2003) untersuchen die Merkmale und Voraussetzungen des Stakeholderdialogs im Vergleich zu stakeholder debate und präsentieren vier verschiedene Formen des Stakeholderdialogs: A Broad, Proactive Dialogue, Stakeholder Panel, Selective Reactive Stakeholder Dialogue und Defensive Dialogue. Louoma-aho und Vos (2010) erläutern u.a. die dynamische Natur der für Stakeholder (-dialoge) relevanten issues und die daraus erforderliche ständige Aufmerksamkeit seitens des Unternehmens. 
gen Dialog, obwohl der Dialog und dessen Darstellung immer mit der Abwägung zwischen Offenheit und Vorsicht bzw. Geheimhaltung verbunden bleiben wird.

Im Anschluss an die oben angeführten Perspektiven auf den Stakeholderbegriff lassen sich in Bezug auf die Interaktion und Kommunikation mit den Stakeholdern drei Handlungsansätze unterscheiden, die im nächsten Abschnitt beschrieben werden.

\subsubsection{Der Stakeholderinformationsansatz}

Dieser Ansatz wird hauptsächlich als Einwegkommunikation vom Unternehmen an die Stakeholder realisiert. Das Unternehmen versorgt seine Stakeholder mit relevanten Informationen und geht davon aus, dass seine Handlungen und Entscheidungen durch rechtzeitiges Informieren bei den Empfängern Akzeptanz, Sympathie und Vertrauen erzeugen. Demnach soll durch die Kommunikation hauptsächlich informiert statt überzeugt werden und es wird angenommen, dass die Meinungen und Reaktionen der Stakeholder zu Gunsten des Unternehmens ausfallen, weshalb man sich weniger um die Ermittlung von Stakeholdermeinungen kümmert. Die Stakeholder werden zwar als einflussreiche Akteure betrachtet, die Widerstand leisten können, aber wegen der moralischen Unantastbarkeit und gesellschaftlichen Relevanz des Unternehmens lassen sich Boykotts und Ähnliches verhindern, wenn hinreichend und konsistent informiert wird (vgl. Morsing/Schultz 2006).

\subsubsection{Der Stakeholderresponsansatz}

Der Stakeholderresponsansatz manifestiert sich als Zweiwegkommunikation zwischen Unternehmen und Stakeholdern, die allerdings nicht als gleichwertige Kommunikationspartner betrachtet werden. Dieses asymmetrische Verhältnis zu Gunsten des Unternehmens bewirkt, dass die Unternehmenskommunikation einen stärker persuasiven Zweck hat als beim Stakeholderinformationsansatz. Die Reaktion der Stakeholder spielt eine entscheidende Rolle für die Planung und Gestaltung der Unternehmenskommunikation und es wird großer Wert darauf gelegt, die Stakeholder von den Qualitäten des Unternehmens zu überzeugen und den Erfolg hierbei zu messen. Die Stakeholder werden als einflussreiche, aber passive Empfänger angesehen, deren Erwartungen durch Befragungen erfüllt werden können. Wenn Fragen an die Stakeholder ausschließlich in einem vom Unternehmen festgelegten Rahmen formuliert werden, liegt jedoch eine Gefahr der Begrenzung darin, dass alternative Fragen möglicherweise nicht erfasst werden, weil sie im Unternehmen übersehen oder nicht für relevant gehalten werden (vgl. Morsing/Schultz 2006).

\subsubsection{Der Stakeholderinvolvierungsansatz}

Im Gegensatz zu den beiden vorigen Ansätzen basiert der Stakeholderinvolvierungsansatz auf einer symmetrischen Beziehung zwischen dem Unternehmen und seinen Stakeholdern. Dialog, laufende Verhandlungen, gegenseitige Beeinflussung und Überzeugung sind zentrale Elemente der Stakeholderinvolvierung. Voraussetzung ist, dass beide Seiten Einsicht in die Haltungen der anderen Seite gewinnen und bereit sind, das eigene Verhalten in Frage zu stellen und zu ändern. Die Stakeholder werden als einflussreiche Befürworter oder Gegner betrachtet und auch bei diesem Ansatz werden 
Meinungsumfragen vorgenommen. Diese werden aber um eine umfassendere Involvierung der Stakeholder ergänzt (vgl. Morsing/Schultz 2006).

Zusammenfassend ist festzuhalten, dass die drei beschriebenen Ansätze dem Dialog mit Stakeholdern recht unterschiedliche Bedeutung zuschreiben, was sich auch in der schriftlichen Unternehmenskommunikation widerspiegelt. Es wird deutlich, dass der Stakeholderinvolvierungsansatz der in 2.1.2 herangezogenen Definition von Dialog am meisten entspricht.

\section{Darstellung von Dialogorientierung und Stakeholderdialog in Geschäftsberichten}

Im Folgenden wird erläutert, wie der Stakeholderdialog in der Unternehmenskommunikation zum Ausdruck kommt, um die oben skizzierten Richtlinien und Empfehlungen zu erfüllen. Geschäftsberichte von fünf deutschen ${ }^{11}$ und fünf dänischen $^{12}$ exemplarisch ausgewählten Chemieunternehmen für die Berichtsjahre 2008 und 2009 wurden analysiert. Es wurden Unternehmen der Chemiebranche gewählt, weil sie durch ihre Produktion und Produkte große gesellschaftliche Aufmerksamkeit erlangen und zunehmend kritischen Blicken ausgesetzt sind. Es ist davon auszugehen, dass der Bedarf an Stakeholderdialog und Kommunikation darüber in der Chemiebranche dringender ist als in vielen anderen Branchen.

Anhand der folgenden drei Fragestellungen wurde die Darstellung des Stakeholderdialogs in den Geschäftsberichten ausgewertet:

(1) In welchem Umfang wird der Stakeholderdialog dargestellt und welche Arten von Information werden über den Stakeholderdialog (nicht) gegeben?

(2) Wie wird der Stakeholderdialog sprachlich evaluiert?

(3) Welche der drei oben beschriebenen Ansätze zum Stakeholderdialog kommen direkt oder indirekt zum Ausdruck?

Unter Anwendung textanalytischer Methoden sollen Antworten auf diese Fragen einen Einblick in die kommunikative Umsetzung des Stakeholderdialogs gewähren. Die hier angelegte sprachwissenschaftliche Perspektive basiert auf einer sozialkonstruktivistischen Grundlage und fokussiert auf die (sozialen) Funktionen der angewandten Sprache in der Relation zwischen Sender und Empfänger. So werden z. B. Aussagen über die (Selbst-) Darstellung und die mögliche Einwirkung auf die Leser durch Belege im Sprachgebrauch untermauert.

\subsection{Umfang und Art der Darstellung des Stakeholderdialogs}

Als ein erster Indikator für den kommunikativen Umgang mit dem Stakeholderdialog wurde die Verwendung des Wortes „Dialog“ quantitativ erfasst. In der Abbildung 1 wird dargestellt, wie oft der Begriff explizit verwendet wird. Die Anzahl wurde jeweils durch eine elektronische Auszählung der Online-Versionen der Geschäftsberichte für

\footnotetext{
11 BASF, Bayer, Evonik Industries, Henkel, Nanogate.

12 Auriga, Bavarian Nordic, Dyrup, Lundbeck, Novo Nordisk.
} 
2008 und 2009 ermittelt. Hierdurch kann erstens festgestellt werden, dass der Begriff und damit auch das Thema Dialog tatsächlich in allen untersuchten Geschäftsberichten vorkommt und zweitens, dass die Spannbreite recht groß ist. Sie reicht von 41 Nennungen bei BASF im Berichtszeitraum 2009 bis zu lediglich einer einzelnen Erwähnung bei Auriga (2009) und Dyrup (2009). Die Aufzählung erfasst nicht eventuell vorkommende Synonyme des gesuchten Begriffs wie beispielsweise Gespräch, Besprechung, Kontakt, Austausch, weil diejenigen Konnotationen und Assoziationen, die mit Dialog verbunden werden, sich nicht automatisch auch an die Synonyme knüpfen. Sie haben deshalb nicht den gleichen Stellenwert bzw. die gleiche Wirkung.

\begin{tabular}{|l|c|c|}
\hline \multicolumn{1}{|c|}{ Unternehmen } & $\begin{array}{c}\text {,Dialog' } \mathbf{~ i m ~ G B ~} \\
\mathbf{2 0 0 8}\end{array}$ & $\begin{array}{c}\text {,Dialog' } \mathbf{~ i m ~ G B ~} \\
\mathbf{2 0 0 9}\end{array}$ \\
\hline BASF (de) & 34 & 41 \\
\hline Bayer (de) & 3 & 3 \\
\hline Evonik (de) & 3 & 4 \\
\hline Henkel (de) & 3 & 6 \\
\hline Nanogate (de) & 2 & 2 \\
\hline Auriga (dk) & 2 & 1 \\
\hline Bavarian Nordic (dk) & 10 & 6 \\
\hline Dyrup (dk) & 3 & 1 \\
\hline Lundbeck (dk) & 3 & 6 \\
\hline Novo Nordisk (dk) & 7 & 11 \\
\hline
\end{tabular}

Abbildung 1: Häufigkeiten des Wortes 'Dialog' in fünf deutschen (de) und fünf dänischen (dk) Geschäftsberichten in den Jahren 2008 und 2009 (eigene Quelle)

Die unterschiedlichen Häufigkeiten lassen sich zum Teil dadurch erklären, dass die beiden Unternehmen BASF und Novo Nordisk ihre CSR-Kommunikation in den Geschäftsbericht integriert haben. So hat z. B. der Henkel-Konzern in seinem Nachhaltigkeitsbericht von 2009 dem Stakeholderdialog zwei Seiten gewidmet. Die CSRKommunikation außerhalb des Geschäftsberichts ist in dieser Studie jedoch nicht enthalten, weil der Geschäftsbericht als ein vollständiges, gesetzlich geregeltes, zentrales Dokument betrachtet wird, das auch ohne ergänzende Berichte aussagefähig sein muss. Hinzu kommt, dass auch eine integrierte bzw. nicht-integrierte Berichterstattung Rückschlüsse auf die Priorisierung des Unternehmens nahe legt.

Die zur Verfügung gestellten Informationen über den Dialog mit Stakeholdern beziehen sich sowohl in den deutschen als auch in den dänischen Geschäftsberichten vor allem auf die Dialogpartner und die mit diesen diskutierten Themen, aber auch Angaben zu Zeit und Ort der Dialoge sind mitunter vorhanden. Die erwähnten Dialogpartner sind an erster Stelle Investoren und Finanzanalysten (,Kapitalmarkt'), Mitarbeiter und ihre Vertreter, aber auch direkte Kunden und Produktnutzer, Lieferanten und andere Geschäftspartner, Behörden, Politiker, Journalisten, Nachbarn, Verbände, 
Organisationen, Wissenschaft und Öffentlichkeit werden genannt. Weiter werden internationale Dialogforen wie beispielsweise der UN Global Compact und das World Economic Forum erwähnt. Formulierungen wie ,alle wesentlichen Stakeholder', ,alle unsere Stakeholder' oder als Hinzufügung nach einer Auflistung ,und andere Stakeholder' treten ebenfalls relativ häufig auf. Nur als eine Ausnahme von der durchgängig gruppenweisen Benennung von Stakeholdern werden konkretere Informationen gegeben, so z. B. das Bundesministerium für Umwelt, Naturschutz und Reaktorsicherheit (vgl. BASF 2008: 41). In den deutschen Geschäftsberichten wird darüber hinaus der Dialog zwischen Aufsichtsrat und Vorstand im Bericht des Aufsichtsrats oft betont. Dieser Dialog ist in diesem Zusammenhang zwar nicht als Stakeholderdialog zu betrachten, aber er signalisiert eine Dialogbereitschaft des Topmanagements und dass diese als nennenswert eingeschätzt wird.

Die im Stakeholderdialog besprochenen Themen sind vielfältig: neue Technologien, vor allem Chancen und Risiken der Nano- und Biotechnologien, der demografische Wandel in Europa, Klimaschutz, Energieeffizienz, Chemikalienpolitik, Umweltschutz und Nachhaltigkeit. Im Dialog mit dem Kapitalmarkt sind Forschungsergebnisse, Ziele und Strategien des Unternehmens besonders relevant. Über den Dialog mit den Mitarbeitern werden vorwiegend Themen wie Arbeitssicherheit, Schulungen, Förderung und Weiterentwicklung sowie Unternehmenswerte angegeben.

Worüber der Leser der Geschäftsberichte wenig erfährt, ist, zu welchen konkreten Ergebnissen die Dialoge geführt haben und welchen Einfluss sie auf die Entscheidungen des Managements (gehabt) haben. Ausnahmen sind bei BASF und Novo Nordisk zu finden, die allerdings auch nicht auf Details eingehen, aber einen Eindruck von Beeinflussbarkeit vermitteln: „Der Austausch mit ihnen [den Stakeholdern] hilft uns, wichtige Themen und daraus folgende Chancen und Risiken für BASF zu erkennen. Die Ergebnisse des Stakeholder-Dialogs fließen in unser Themenmanagement für Nachhaltigkeit ein" (BASF 2009: 29) und ,when setting the target [for CO2 reduction], we shared internal data with WWF and had a very open dialogue. WWF challenged us to set the bar higher than we would have otherwise done" (Novo Nordisk 2009: 33, eng. Version).

\subsection{Sprachliche Evaluierung des Stakeholderdialogs}

Dieser Aspekt bezieht sich auf den bewertenden Sprachgebrauch, hierunter die gewählten Verben und Akteure, Adjektive, Pronomen und rhetorischen Mittel zur Beschreibung des Stakeholderdialogs. Kennzeichnend für die gewählten Verben ist, dass sie positiv konnotierte Handlungen beschreiben, die von Tatkraft, Initiative und Engagement zeugen. Das Unternehmen wird dabei als Akteur dargestellt, oft durch das persönlicher wirkende und distanzreduzierende Personalpronomen, wir‘. Beispiele hierfür aus den deutschen Berichten: „Unsere Mitarbeiter binden wir in den Dialog um Nanotechnologien mit ein“ (BASF 2008: 41); ,im internationalen Dialog engagieren wir uns ..." (Bayer 2008: 102); „Daher haben wir uns ...intensiv in den nationalen und internationalen Dialog darüber eingebracht" (Henkel 2009: 57). Ähnliche Konstruktionen kommen auch in den dänischen Berichten vor, aber häufig sind hier auch modifizierende Ausdrücke (möchten, erwarten, hoffen, versuchen) angewandt wie z. B. ,Jeg forventer, at vores dialog med interessenterne intensiveres, og jeg håber, vi 
kan skabe en større forståelse for, hvad det er, vi prøver at opnå“ („Ich erwarte, dass unser Dialog mit den Interessenten intensiviert wird und ich hoffe, dass wir ein größeres Verständnis dafür schaffen können, was es ist, dass wir versuchen zu erreichen“, Novo Nordisk 2009: 8; übersetzt durch d. V.). Auf der einen Seite wird hierdurch weniger Sicherheit signalisiert, aber auf der anderen Seite wird auch die gegenseitige Abhängigkeit im Dialog impliziert.

Wie aus den obigen Beispielen hervorgeht, werden in den sprachlichen Konstruktionen, deutschen wie dänischen, sehr oft auch Präsuppositionen eingebaut, die den Dialog positiv evaluieren und als Fakt darstellen, dass der Dialog schon länger stattfindet, z. B.: "Der Dialog mit Investoren, die ihre Anlageentscheidungen wesentlich an Nachhaltigkeitskriterien orientieren, wurde bei Konferenzen und in Einzelgesprächen weiter intensiviert" (Bayer 2008: 20). Allein das häufig benutzte Verb ,intensivieren“ und Adverbien wie „weiter“, „,weiterhin“ und „erneut" deuten an, dass der Stakeholderdialog zur geübten Praxis des Unternehmens gehört.

Auch in der Wahl der charakterisierenden Adjektive scheinen bestimmte Wörter bevorzugt zu werden. Erstens zur Betonung der Regelmäßigkeit des Dialogs, etwa: „Der jährliche Dialog ...“ (Evonik 2009: 78) und „Im regelmäßigen Dialog ...“ (Bayer 2009: 111). Zweitens werden die Adjektive „gut“, „offen“, „eng“ und ,intensiv“ in beiden Sprachen häufig zur Aufwertung des Dialogs verwendet. Problematisch an dieser Art Beschreibung ist aber, dass sie als subjektive Bewertung ohne nähere Erklärung dasteht und deshalb für den Leser wenig informativen Wert hat. Gleichzeitig wird hiermit indirekt darauf hingewiesen, dass ein qualitativ so hochwertiger Dialog wie vom jeweiligen Unternehmen geführt nicht selbst-verständlich ist - der Dialog könnte schließlich auch schlecht, verschlossenen, distanziert oder wenig intensiv ausfallen.

Alles in allem hinterlassen die Darstellungen einen Eindruck von sehr dialogbereiten Unternehmen, die sich nicht zum Stakeholderdialog gezwungen fühlen, sondern den Dialog mit ihren Stakeholdern proaktiv und gerne suchen. Der hier untersuchte Sprachgebrauch hat mit dem Sprachgebrauch in Newsletters für Investoren große Ähnlichkeit (vgl. Pedersen 2008).

\subsection{Dokumentierte Stakeholderdialogansätze}

Die drei oben beschriebenen Stakeholderdialogansätze können in den Geschäftsberichten in unterschiedlichem Umfang identifiziert werden. Vor allem der Stakeholderinformationsansatz und der Stakeholderresponsansatz treten in den untersuchten Texten auf. Der Stakeholderinformationsansatz scheint jedoch der dominanteste zu sein. Die Unternehmen erwähnen an verschiedenen Stellen, dass sie informieren, orientieren und Informationen (im Internet) zur Verfügung stellen, so etwa: „Vorstand und Investor-Relations-Abteilung absolvierten mehr als 400 Einzelgespräche an rund 25 Finanzplätzen und informierten über aktuelle Themen des Konzerns (Bayer 2008: 20); „Um die Informationswünsche unserer Stakeholder zielgruppengerecht bedienen zu können, nutzen wir eine Vielzahl von Kommunikationsinstrumenten“ (Henkel 2008: 57). Hier wird explizit unterstellt, dass die Stakeholder (nur) Informationen wünschen. Konferenzen, Roadshows, Präsentationen und Einzelgespräche werden in vielen Fällen als Beispiele für den Dialog mit Investoren dargestellt. Im Geschäftsbe- 
richt der BASF AG (2009: 10f.) wird auch eine visuelle Strategie benutzt, um Dialogorientierung zu signalisieren und den Stakeholderdialog zu illustrieren. Ein doppelseitiges Bild zeigt den Vorstand im Dialog mit Mitarbeitern. Die in weißen und hellblauen Hemden, ohne Jacken und Krawatten gekleideten Vorstandsmitglieder stehen mit offener, entgegenkommender Körpersprache und Lächeln im oberen Hintergrund des Bildes; sie stehen hinter hellen, offenen Theken und schauen auf die Mitarbeiter. Die Dialogpartner sind jedoch nicht auf gleicher Augenhöhe dargestellt, da der Vorstand auf einem Podium zu stehen scheint und ihre Blickrichtung nach unten geht.

Der Stakeholderresponsansatz spiegelt sich am deutlichsten in den Berichten von BASF, Evonik und Novo Nordisk wider. Befragungen von vor allem Mitarbeitern (vgl. Evonik 2009: 78) und Lesern (vgl. BASF 2008: 2) werden erwähnt und positiv bewertet. Bei Novo Nordisk (2008: 119; 2009: 93) wird Responsiveness als Teil der Accounting policies genannt, wobei die Leser zu Rückmeldungen aufgefordert werden: "Stakeholder dialogue is an invaluable part of Novo Nordisk's efforts as a responsible business, and readers are encouraged to give their feedback". Allerdings ist es nicht unproblematisch, wenn das Unternehmen den Eindruck vermittelt, dass die Reaktion von allen Stakeholdern (gleich) wichtig ist, so z. B.: „We create results for our customers by being a sustainable and innovative world-class supplier of a broad range of quality crop protection products. Value creation shall match the best among peer companies to the benefit of all stakeholders" (Auriga 2008: 4). Betont wird also die Wertschaffung zu Gunsten aller Stakeholder - wie das praktisch umgesetzt wird, bleibt aber unklar. Möglicherweise fühlen sich Kunden nicht berücksichtigt, wenn sie auf der nächsten Seite im Bericht lesen, dass es dem Unternehmen gelungen sei, Preiserhöhungen am Markt durchzusetzen, denn höhere Preise sind kaum im Interesse der Kunden. Dieses Beispiel, das keineswegs einzigartig ist, verdeutlicht das Unmögliche, allen Stakeholdern, gefallen' zu wollen. Bemerkenswert ist auch die in Nanogate (2008: 11) angedeutete Unzufriedenheit mit der (ausgebliebenen) Reaktion der Empfänger zu ihrer Finanzkommunikation: „Die erfreuliche Unternehmens-entwicklung mit kontinuierlich steigender Geschäftstätigkeit, neuen Projekten und erweiterter Marktpräsenz mit zusätzlichen Kunden spiegelt sich trotz intensiver Finanzkommunikation nicht im Aktienkurs wider".

In vagen Formulierungen taucht auch der Stakeholderinvolvierungsansatz sporadisch auf, indem einerseits der Wert der Einbeziehung von Stakeholdern hervorgehoben und andererseits die grundlegende Verankerung der aktiven Pflege der Stakeholderbeziehungen herausgestellt wird. Dies zeigt sich etwa in folgender Formulierung: „Zukünftige Lösungen für ein nachhaltiges Wirtschaften können nur im Dialog mit allen gesellschaftlichen Gruppen entwickelt werden“ (Henkel 2008: 57). Erneut sehr deutlich ist die Darstellung der BASF AG: „Der Austausch mit ihnen [den Stakeholdern] hilft uns, wichtige Themen und daraus folgende Chancen und Risiken für BASF zu erkennen" (BASF 2008: 29). Hieraus ergibt sich auch explizit der Nutzen für das Unternehmen, was skeptische Leser einerseits vom Wert des Stakeholderdialogs für das Unternehmen, andererseits aber auch von der Ernsthaftigkeit des Dialogs überzeugen soll. 


\subsection{Zusammenfassende Bewertung bisheriger Berichterstattung}

Zusammenfassend kann festgestellt werden, dass die Unternehmen in ihren Geschäftsberichten in sehr unterschiedlichem Umfang ein dialogorientiertes Selbstbild präsentieren. Wenn aber Inhalt und Formulierungen näher betrachtet werden, unterscheiden sich die Selbstbilder nicht sehr voneinander, weil wenig unternehmensspezifische, konkrete Auskünfte gegeben werden. Die integrierte Berichterstattung der Unternehmen BASF und Novo Nordisk unterscheidet sich jedoch durch die häufigere Nennung des Begriffs ,Dialog', die deutlichere Signalisierung der eigenen Beeinflussbarkeit und des aus dem Stakeholderdialog erzielten Nutzens, den dargestellten Stakeholderresponsansatz und, bei BASF, die Visualisierung des Stakeholderdialogs. Dieses Ergebnis legt die Interpretation nahe, dass die Integration der Nachhaltigkeitsberichterstattung in die Geschäftsbericht-erstattung mit einer intensiveren Darstellung des Stakeholderdialogs verbunden ist. Wenn es zur Wichtigkeit und Relevanz der in den untersuchten Geschäftsberichten genannten Stakeholder kommt, werden den Stakeholdergruppen Investoren, Finanzanalysten und Mitarbeiter (-vertretern) die größte Relevanz und Wichtigkeit zugeschrieben (Abschnitt 3.1). Aus einer normativen Betrachtung des Stakeholderbegriffs (Abschnitt 2.1.1) müssten aber die übrigen Stakeholdergruppen wie beispielsweise Geschäftspartner, Behörden und Öffentlichkeit intensiver einbezogen werden, um die Basis der Unternehmen für gesellschaftliche Unterstützung auszubauen. Der Dialogbegriff scheint in vielen Fällen mehr als Schlagwort benutzt zu werden denn als Ausdruck einer eigentlichen dialogischen Praxis zu sein. Das schließt aber nicht aus, dass diese tatsächlich stattfindet. Dabei stellt sich die Frage nach den Funktionen der dialogorientierten Selbstdarstellung. Bezweckt sie allein die Erfüllung der gegebenen Richtlinien oder soll sie auch der Vermittlung von Corporate Identity und der Positionierung unter Mitbewerbern dienen? Aus Sicht der Verfasserin besteht das Potenzial, beides zu erreichen, was im nächsten Abschnitt näher erläutert wird.

\section{Ungenutzte Potenziale vom Stakeholderdialog als Differenzierungsmerkmal}

Greift man auf die oben beschriebenen Merkmale von Dialog (Abschnitt 2.1.2) zurück und bedenkt, dass ein Dialog viel mehr und viel anspruchsvoller ist als ein normales Gespräch, werden Diskrepanzen zwischen diesen Merkmalen und den dargestellten Stakeholderdialogen deutlich. Auch wenn der idealisierte Dialog in der Unternehmenspraxis nicht vollständig realisierbar ist, bleiben wichtige Potenziale ungenutzt, die für die Leser und somit auch für die Unternehmen einen größeren Nutzen haben könnten. Gerade weil der Dialog anspruchsvoll ist, indem er Offenheit und Mut erfordert, und gleichzeitig Zugang zu einer Horizonterweiterung gibt, kann eine nuanciertere und informativere Darstellung als Differenzierungsmerkmal eingesetzt werden. Mehrere Argumente sprechen dafür:

- Stakeholder sind zu einem gewissen Grad unternehmensspezifisch. Damit ist gemeint, dass sich die Stakeholder je nach Produkt, Marktposition, Standort und ähnlichen Faktoren unterscheiden. Beispielsweise gehören Patientenvereine zu den Stakeholdern der meisten Pharma- und Medizintechnikunternehmen, was nicht bei Autoherstellern der Fall ist. Die Mitarbeiter, die Investoren, die 
Nachbarn usw. sind also für das jeweilige Unternehmen einzigartig und deshalb müssen sich unterschiedliche Ergebnisse aus den Dialogen ergeben, die als solche dargestellt werden können.

- Aus der Perspektive eines Investors, der zur wichtigsten Zielgruppe des Geschäftsberichts gehört, wäre vermutlich interessant und relevant zu wissen, mit wem das Unternehmen einen Dialog führt und welchen Einfluss die verschiedenen Dialogpartner auf die Geschäftstätigkeit des Unternehmens haben. Auch aus Sicht anderer Stakeholder scheint es nicht unwichtig zu sein, ob der Stakeholderdialog beispielsweise zu Änderungen im Produktions-verfahren oder in den Prioritäten des Managements geführt hat.

- $\quad$ Eine konkretere und differenziertere Darstellung des Stakeholderdialogs würde ein besseres Verständnis der Unternehmensidentität bedeuten, was oft eine schwierige kommunikative Herausforderung ist.

- Zur Risikoeinschätzung inner- und außerhalb des Unternehmens wäre eine konkrete Darstellung der relevanten Stakeholder, der Dialogpraxis und der Ergebnisse nützlich. Denn es kann als Risiko betrachtet werden, wenn eine Stakeholdergruppe für das Unternehmen kontraproduktive Handlungen initiiert. Je konkretere und detailliertere Auskünfte zur Verfügung stehen, desto größer ist möglicherweise das Gefühl von Sicherheit auf Seiten der Investoren.

Sicherlich ist der Grad an Konkretisierung mit einem Abwägen zwischen Geheimhaltung von Geschäftsgeheimnissen und Vertraulichkeit auf der einen Seite und Transparenz und Differenzierung auf der anderen Seite verbunden. Was glaubwürdiger ist, wird wahrscheinlich von den verschiedenen Zielgruppen unterschiedlich bewertet.

\section{Abschließende Bemerkungen}

Betrachtet man die in diesem Beitrag untersuchte Darstellung des Stakeholderdialogs aus der Perspektive der Corporate Governance Regulierungen, scheinen sämtliche Unternehmen diese zu erfüllen, indem berichtet wird, dass Stakeholderdialoge stattfinden. Aus der Perspektive der Profilierung und Differenzierung sind aber im Sprachgebrauch und in der Selektion von Informationen Verbesserungen möglich. Es scheint eine Diskrepanz zwischen der erklärten Offenheit und Transparenz und der in den Texten gezeigten Offenheit und Transparenz zu geben. Im sprachlichen Umgang mit Dialog und Dialogorientierung in der Unternehmenskommunikation wie in der Literatur über Unternehmenskommunikation scheint weitgehend vorausgesetzt zu werden, dass jeder weiß, was mit Dialog gemeint ist, und dass es deshalb keiner näheren Erklärung bedarf. ,Dialog' wird als bu₹zword verwendet und über Texte gestreut ohne eine tatsächliche Auseinandersetzung mit Bedeutung, Funktion und zentralen Merkmalen zu signalisieren.

\section{Literaturverzeichnis}

Auriga Industries A/S (2009): Auriga Årsrapport 2009, online unter: http://www.auriga.dk/dk/ regnskab/aarsrapporter/2009.htm, Zugriff am: 27.05.2011.

Auriga Industries A/S (2008): Auriga Årsrapport 2008, online unter: http://www.auriga.dk/dk/ regnskab/aarsrapporter/2009.htm, Zugriff am: 26.05.2011. 
BASF AG (2009): BASF Bericht 2009: Ökonomische, ökologische und gesellschaftliche Leistung, online unter: http://www.basf.com/group/corporate/de/investor-relations/ news-publications/reports/index, Zugriff am: 26.05.2011.

BASF AG (2008): Wir gestalten Zukunft. Bericht 2008: Ökonomische, ökologische und gesellschaftliche Leistung, online unter: http://www.basf.com/group/corporate/de /investor-relations/news-publications/reports/index, Zugriff am: 26.05.2011.

Bassen, A./ Jastram, S./ Meyer, K. (2005): Corporate Social Responsibility. Eine Begriffserläuterung, in: Zeitschrift für Wirtschafts- und Unternehmensethik, Jg. 6/ Heft 2, 231-236.

Bauhofer, B. (2004): Reputation Management. Glaubwürdigkeit im Wettbewerb des 21. Jahrhunderts, Zürich, Orell Füssli Verlag.

Bavarian Nordic A/S (2009): Årsrapport 2009, online unter: http://www.bavarian-nordic.com/ investor/financials.aspx, Zugriff am: 27.05.2011.

Bavarian Nordic A/S (2008): Årsrapport 2008, online unter: http://www.bavarian-nordic.com/ investor/financials.aspx, Zugriff am: 27.05.2011.

Bayer AG (2009): Geschäftsbericht 2009, online unter: http://www.investor.bayer.de/berichte, Zugriff am: 26.05.2011.

Bayer AG (2008): Geschäftsbericht 2008, online unter: http://www.investor.bayer.de/berichte, Zugriff am: 26.05.2011.

Beck, U./ Hajer, M./ Kesselring, S. (Hrsg.) (1999): Der unscharfe Ort der Politik, Opladen: Leske und Budrich.

Beck, U. (2003): World Risk Society, Cambridge: Polity Press.

Bextermöller, M. (2006): Über das Geschäft schreiben. Mit Ausdruckskraft zu überzeugenden Geschäftsberichten, Paderborn: Verlag Peter Ewers.

Bloch-Poulsen, J. (2006): Dialog - En Udfordring: Mellem Involvering og Demokratur I Organisationsudvikling, Aalborg: Aalborg Universitetsforlag.

Braun, S. (2008): Gesellschaftliches Engagement von Unternehmen in Deutschland, in: Aus Politik und Zeitgeschichte, Heft 31, 6-14.

Brock, D. (2008): Globalisierung. Wirtschaft - Politik - Kultur - Gesellschaft, Wiesbaden: VS Verlag für Sozialwissenschaften.

Burchell, J./ Cook, J. (2006): Assessing the impact of stakeholder dialogue: changing relationships between NGOs and companies, in: Journal of Public Affairs, Jg. 6/ Heft 3-4, 210-227.

Burchell, J./ Cook, J. (2008): Stakeholder dialogue and organisational learning: changing relationships between companies and NGOs, in: Business Ethics: A European Review, Jg. 17/ Heft 1, 35-46.

Deix, G. (2005): Ethische Grundlagen von Unternehmenskommunikation und Stakeholderdialog, in: Brink, A./ Tiberius, V. A. (Hrsg.): Ethisches Management. Grundlagen eines wert(e)orientierten Führungskräfte-Kodex, Bern/Stuttgart/Wien: Haupt Verlag, 333378.

Dresewski, F./ Koch, S.C. (2011): Zusammenarbeit von Unternehmen und zivilgesellschaftlichen Akteuren - Überblick und Fallbeispiele, in: Raupp, J./Jarolimek, S./ Schultz, F. (Hrsg.): Handbuch CSR - Kommunikationswissenschaftliche Grundlagen, disziplinäre Zugänge und methodische Herausforderungen, Wiesbaden: VS Verlag, 450-460.

Dyrup A/S (2009): Dyrup A/S - Årsrapport 2009, online unter: http://www.dyrup.com/ Finance/Annual\%20Report\%202009.aspx, Zugriff am: 27.05.2011.

Dyrup A/S (2008): Dyrup A/S - Årsrapport 2008, online unter: http://www.dyrup.com/ Finance/Annual\%20Report\%202008.aspx, Zugriff am: 27.05.2011. 
Evonik Industries AG (2009): Geschäftsbericht 2009. Ideenland. Eine Reise zu den Lösungen für morgen, online unter: http://corporate.evonik.de/de/investoren/publikationen/ pages/default.aspx, Zugriff am: 26.05.2011.

Evonik Industries AG (2008): Geschäftsbericht 2008. Die Welt braucht Ideen, online unter: http://corporate.evonik.de/de/investoren/publikationen/pages/default.aspx, Zugriff am: 26.05.2011.

Frederiksen J./ Honoré, C./ Tost, F. (2003): Rapportering om bæredygtighed, Copenhagen: Børsen.

Freeman, R. E. (1984): Strategic Management. A Stakeholder Approach, Marshfield, MA: Pitman.

Henderson, D. (2001): Misguided Virtue. False Notions of Corporate Social Responsibility, London: The Institute of Economic Affairs.

Henkel $A G$ \& Co. KGaA (2009): Globales Team - gemeinsam gewinnen. Geschäftsbericht 2009, online unter: http://www.henkel.de/investor-relations/geschaeftsbericht10402.htm, Zugriff am: 26.05.2011

Henkel $A G$ \& Co. KGaA (2008): Geschäftsbericht 2008 - Den Wandel aktiv gestalten, online unter: http://www.henkel.de/investor-relations/geschaeftsbericht-10402.htm, Zugriff am: 26.05.2011.

Hiß, S. (2009): Corporate Social Responsibility - Innovation oder Tradition? Zum Wandel der gesellschaftlichen Verantwortung von Unternehmen in Deutschland, in: Zeitschrift für Wirtschafts- und Unternehmensethik, Jg. 10/ Heft 3, 287-303.

Holmgreen, L. (2008): Communicating Financials in the Biotech Industry: A Strategic Decision?, in: Dam, L./ Holmgreen, L./ Strunck, J.(Hrsg.): Rhetorical Aspects of Discourses in Present-Day Society, Newcastle upon Tyne: Cambridge Scholars Publishing, 359-373.

Kaptein, M./ van Tulder, R. (2003): Toward Effective Stakeholder Dialogue, in: Business and Society Review, Jg. 108/ Heft 2, 203-224.

Kinder, P.D./ Lydenberg, S.D./ Domini, A.L. (1992): The Social investment almanac: a comprehensive guide to socially responsible investing, New York: H. Holt and Co.

Kommission der Europäischen Gemeinschaften (2001): Grünbuch - Europäische Rahmenbedingungen für die soziale Verantwortung der Unternehmen, KOM (2001) 366 endgültig, Brüssel, online unter: http://eur-lex.europa.eu/LexUriServ/site/de/ com/2001/ com2001_0366de01.pdf, Zugriff am: 26.05.2011.

Larsen, A. V./ Pedersen, A. G. J. (2010): Klimakommunikation von Fluggesellschaften - eine empirische Untersuchung der Sender-Empfänger-Relationen in der OnlineKlimakommunikation von deutschen und dänischen Fluggesellschaften, in: Kvam, S./ Knutsen, K. P./ Langemeyer, P. (Hrsg.): Textsorten und Kulturelle Kompetenz - Genre and Cultural Competence. Interdisziplinäre Beiträge zur Textwissenschaft - An Interdisciplinary Approach to the Study of Text, Münster: Waxmann.

Lindgreen, A./ Swaen, V. (2010): Corporate Social Responsibility, in: International Journal of Management Reviews, Jg. 12/ Heft 1, 1-7.

Lozano, J.M. (2005): Towards the relational corporateion: from managing stakeholder relationships to building stakeholder relationships (waiting for Copernicus), in: Corporate Governance, Jg. 5/Heft 2, 60-77.

Lundbeck A/S (2009): Årsrapport 2009, online unter: http://www.lundbeck.com/investor/ Financials/Reports/default.asp, Zugriff am: 27.05.2011.

Lundbeck A/S (2008): Årsrapport 08, online unter: http://www.lundbeck.com/investor/Financials/Reports/default.asp, Zugriff am: 27.05.2011. 
Luoma-aho, V./ Vos, M. (2010): Towards a more dynamic stakeholder model: acknowledging multiple issue arenas, in: Corporate Communications: An International Journal, Jg. 15/ Heft 3, 315-331.

May, S./ Cheney, G./ Roper, J. (2007): The debate over corporate social responsibility, Oxford University Press.

Morsing, M. (2005): CSR - Et organisationsudviklingsprojekt, in: KOM Magasinet - Magasinet for kommunikation og sprog, Heft 8, 12-13.

Morsing, M. / Schultr, M. (2006): Corporate social responsibility communication: stakeholder information, response and involvement strategies, in: Business Ethics: a European Review, Jg. 15/ Heft. 4, 323-338.

Nanogate AG (2009): Geschäftsbericht 2009 - Innovations-Partnerschaft, online unter: http://www.nanogate.de/de/investoren/finanzberichte, Zugriff am: 27.05.2011.

Nanogate AG (2008): Geschäftsbericht 2008 - Wettbewerbsvorsprung durch Innovation, online unter: http://www.nanogate.de/de/investoren/finanzberichte, Zugriff am: 27.05.2011.

Novo Nordisk A/S (2009): novo nordisk årsrapport 2009 - økonomiske, sociale og miljømæssige resultater. Værdiskabelse gennem innovation, online unter: http://www.novonordisk.com/images/annual_report/2009/Novo-Nordisk-AR-2009dk.pdf, Zugriff am: 27.05.2011.

Novo Nordisk A/S (2008): novo nordisk årsrapport 2008 - økonomiske, sociale og miljømæssige resultater. Vores fokus er vores styrke, online unter: http://www.novonordisk.com/images/annual_report/AR_08/Novo_Nordisk_DK_A R2008.pdf, Zugriff am: 27.05.2011.

Pedersen, A.G. (2008): Investor Relations Newsletters. Funktion, Aufbau und Sprache, in: Siems, F. U./ Brandstätter, M./ Gölzner, H. (Hrsg.): Anspruchsgruppenorientierte Kommunikation. Neue Ansätze zu Kunden-, Mitarbeiter- und Unternehmenskommunikation, Europäische Kulturen in der Wirtschaftskommunikation, Wiesbaden: VS Verlag, 371-385.

Raupp, J./ Jarolimek, S./ Schultz, F. (Hrsg.) (2011): Handbuch CSR - Kommunikationswissenschaftliche Grundlagen, disziplinäre Zugänge und methodische Herausforderungen, Wiesbaden: VS Verlag.

Sptizeck, H./ Hansen, E. G. (2010): Stakeholder governance: how stakeholders influence corporate decision making, in: Corporate Governance, Jg. 10/ Heft. 4, 378-391.

van Huijstee, M./ Glasbergen, P. (2007): The Practice of Stakeholder Dialogue between Multinationals and NGOs, in: Corporate Social Responsibility and Environmental Management, Jg. 15/ Heft 5, 298-310.

Wehmeier, S. (Hrsg.) (2000): Oxford Advanced Learner's Dictionary of Current English, 6 ${ }^{\text {th }}$ ed., Oxford University Press.

Wieland, J. (Hrsg.) (2007): Governanceethik und Diskursethik - ein zwangloser Diskurs, Marburg: Metropolis-Verlag.

Zsolnai, L. (Hrsg.): Ethics in the Economy. Handbook of Business Ethics, Bern: Peter Lang. 